Journal of Statistical Research

ISSN 0256 - 422X

2017, Vol. 51, No. 2, pp. 115-129

\title{
ON AN EXTENDED SKEW CURVED NORMAL DISTRIBUTION AND ITS APPLICATION
}

\author{
C. Satheesh KumaR ${ }^{\star}$ \\ Department of Statistics, University of Kerala, Trivandrum 695 581, India \\ Email:drcsatheeshkumar@gmail.com \\ M. R. Anusree \\ Department of Operations, Rajagiri Business School, Kochi 682039, India \\ Email: anusreemr@yahoo.co.in

\section{SUMMARY}

In this paper we introduce a new class of asymmetric normal distributions, namely extended skew curved normal distribution, as a generalization of the skew curved normal distribution of Arellano-Valle et al. (Commun. Statist. Theor. Meth., 2004) and study some of its important aspects. A location scale extension of this family of distribution is also considered and the estimation of the parameters of the extended class is discussed.

Keywords and phrases: Asymmetric distributions, Generalized mixtures, Maximum likelihood estimation, Normal distribution, Plurimodality

\section{Introduction}

The origin of non-symmetric distributions can be traced back to Edgeworth (1886) and Pearson (1893). Interest in asymmetric distributions, in particular, skew normal distribution has been growing tremendously over the past two decades. The first systematic treatment of skew normal distribution was given by Azzalini (1985, 1986), although this idea is suggested in earlier work. For example see Birnbaum (1950), Nelson (1964), Weinstein (1964), Roberts (1966), and ÓHagen and Leonard (1976). Azzalini (1985) defined the skew normal distribution as given below:

A random variable $\mathrm{X}$ is said to follow skew normal distribution with asymmetry parameter $\lambda \in R=(-\infty, \infty)$ if its probability density function (p.d.f.) $g(x ; \lambda)$ is of the following form, in which $x \in R$.

$$
g(x ; \lambda)=2 \phi(x) \Phi(\lambda x),
$$

where $\phi(\cdot)$ and $\Phi(\cdot)$ are respectively the p.d.f. and cumulative distribution function (c.d.f.) of a standard normal variate. The skew normal distribution with p.d.f. (1.1) has been studied extensively in the literature and it has been found application in several areas of

\footnotetext{
* Corresponding author

(c) Institute of Statistical Research and Training, University of Dhaka, Dhaka 1000, Bangladesh
} 
research. For details, see Genton (2004) or the review paper of Azzalini (2005), and the references there in. Arellano-Valle et al. (2004) introduced a generalized form of skew normal distribution namely skew curved normal distribution, through the following p.d.f., in which $x \in R, \lambda \in R$.

$$
g_{1}(x ; \lambda)=2 \phi(x) \Phi\left(\frac{\lambda x}{\sqrt{1+\lambda^{2} x^{2}}}\right)
$$

A distribution with p.d.f. (1.1) we denoted as $S N D(\lambda)$ and a distribution with p.d.f. (1.2) we denoted as $S C N D(\lambda)$. Buccianti(2005) reported that both the normal and the $S N D(\lambda)$ are not capable for describing the situation of plurimodality. He investigated the shape of the frequency distribution of the logratio $\ln (\mathrm{cl}-\mathrm{=} \mathrm{Na}+)$ whose components are related to water composition for 26 wells. Samples have been collected around the active center of Vulcano Island from 1977. Data of the log ratio have been tentatively modeled by evaluating the performance of the skew normal model for each well. Value of the shape parameter say $\lambda$ for wells of Vulcano Island appear to covers a wide range corresponding to a 1) more or less good symmetry, 2) presence of a moderate skewness, 3) presence of plurimodality. For the first and second situation he noted that normal or skew normal model is better and for the third case where these models are not adequate. To accommodate such plurimodal situations, Kumar and Anusree (2011) considered a generalized version of the $S N D(\lambda)$, which they defined as follows:

A random variable $Y$ is said to have a generalized skew normal distribution $\operatorname{GMNSND}(\lambda, \alpha)$ if its p.d.f. is of the following form, in which $y \in R, \lambda \in R$ and $\alpha \geq-1$.

$$
g_{2}(y ; \lambda, \alpha)=\frac{2}{(\alpha+2)} \phi(y)[1+\alpha \Phi(\lambda y)]
$$

A limitation of the $\operatorname{GMNSND}(\lambda, \alpha)$ model is that for moderate values of $\lambda$ nearly all the mass accumulates either on the positive side or on the negative side, as determined by the sign of $\lambda$. In such cases, (1.3) closely resembles the half-normal density, with a nearly linear shape in the side with smaller mass. To partially mitigate such a limitation, through this paper we introduce a new family of asymmetric normal distribution that exhibits a better behavior, particularly at the side with smaller mass. This class of distribution is referred as "the extended skew curved normal distribution $(E S C N D)$ ", as a generalized mixture of the standard normal distribution and the skew curved normal distribution of Arellano-Valle et al. (2004). This interesting property is the basic motivation for considering ESCND. The rest of the paper is organized as follows. In section 2 we present the definition of the $E S C N D$ and discuss some of its properties. In section 3, we derive an explicit expression for moments of the ESCND and in section 4 certain reliability aspects concerning this family is obtained. In section 5 a location scale extension of the ESCND is considered and in section 6, the maximum likelihood estimation of the parameters of the ESCND is discussed. In section 7, a numerical example is given for illustrating the practical usefulness of this new class of distributions and finally, section 8 is the concluding section . 


\section{Extended Skew Curved Normal Distribution}

First we define the $E S C N D$ as in the following.

Definition 2.1. A random variable $Z$ is said to have an extended skew curved normal distribution if its p.d.f. takes the following form, in which $z \in R, \lambda \in R$ and $\alpha \geq-1$.

$$
f(z ; \lambda, \alpha)=\frac{2}{(\alpha+2)} \phi(z)\left[1+\alpha \Phi\left(\frac{\lambda z}{\sqrt{1+\lambda^{2} z^{2}}}\right)\right]
$$

A distribution with p.d.f. (2.1) hereafter we written as $\operatorname{ESCND}(\lambda, \alpha)$. There are several phenomenons which cannot be described by either the normal or the skew normal distributions. In this respect, more flexible probability models near to normal but suitable for both the asymmetric and plurimodal situations are essential. So through this article our main goal is to develop and study certain families of skew normal distributions that is flexible enough to support plurimodal shapes. For particular values of $\alpha$ and $\lambda$, the $\operatorname{ESCND}(\lambda, \alpha)$ reduces to the following special cases.

1. when $\alpha=0$ and or when $\lambda=0$, the $\operatorname{ESCND}(\lambda, \alpha)$ reduces to the standard normal distribution,

2. when $\alpha=-1$, the $\operatorname{ESCND}(\lambda, \alpha)$ reduces to the $\operatorname{SCN} D(-\lambda)$

3. when $\alpha$ tend to $\infty$, the $\operatorname{ESCND}(\lambda, \alpha)$ reduces to $S C N D(\lambda)$

The p.d.f. of the $\operatorname{ESCND}(\lambda, \alpha)$ given in (2.1) is plotted for particular choice of $\lambda$ and $\alpha$ and presented in Figure 1.

Figure 1: Probability plots of $\operatorname{ESCND}(\lambda, \alpha)$ for $\lambda=8.5$ and $\alpha=0.85$

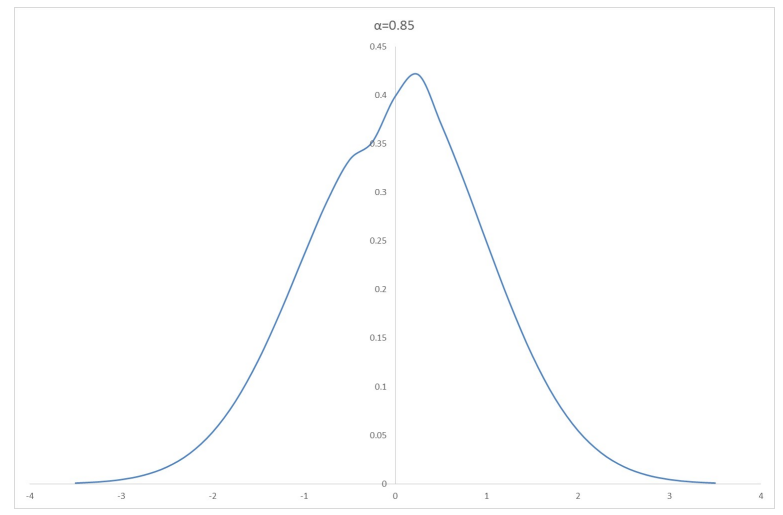

We have also obtained the probability plots of $\operatorname{ESCND}(\lambda, \alpha)$ with the corresponding distributions normal, SND, GMNSND for comparison and is as shown in Figure 2. 
Figure 2: Probability plots of $N, E S N D, S C N D, E G M N S N D$, and ESCND

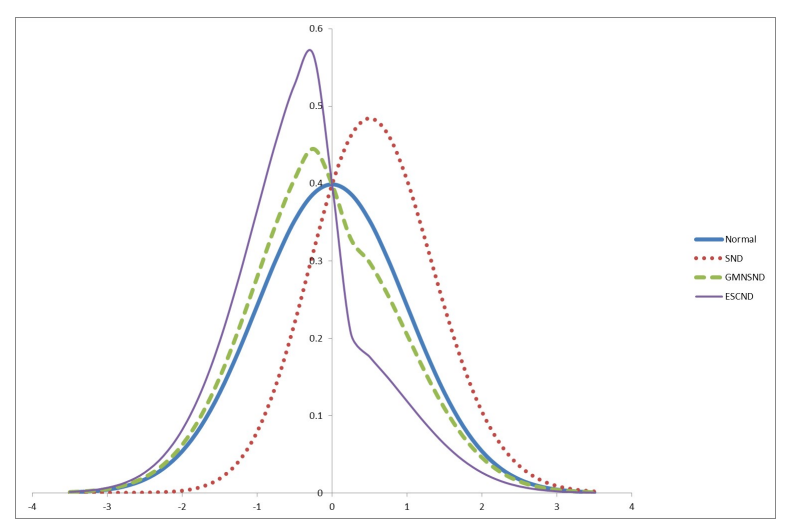

Now we have the following results.

Result 2.1. If $Z$ follows $\operatorname{ESCND}(\lambda, \alpha)$ then $Z_{1}=-Z$ follows $\operatorname{ESCND}(-\lambda, \alpha)$.

Proof. The p.d.f. $f_{1}\left(z_{1} ; \lambda, \alpha\right)$ of $Z_{1}$ is given by

$$
\begin{aligned}
f_{1}\left(z_{1} ; \lambda, \alpha\right) & =f(-z ; \lambda, \alpha)\left|\frac{d z}{d z_{1}}\right| \\
& =\frac{2}{(\alpha+2)} \phi\left(-z_{1}\right)\left[1+\alpha \Phi\left(\frac{-\lambda z_{1}}{\sqrt{1+\lambda^{2} z_{1}^{2}}}\right)\right] \\
& =f\left(z_{1} ;-\lambda, \alpha\right),
\end{aligned}
$$

which implies that $Z_{1}$ follows the $\operatorname{ESCND}(-\lambda, \alpha)$.

Result 2.2. If $Z$ be a random variable following the $\operatorname{ESCND}(\lambda, \alpha)$. Then $|Z|$ follows standard half normal distribution.

Proof. Let $Z_{2}=|Z|$. Then for $z>0$, the p.d.f. $f_{2}\left(z_{2} ; \lambda, \alpha\right)$ of $Z_{2}$ is

$$
\begin{aligned}
f_{2}\left(z_{2} ; \lambda, \alpha\right) & =f(z ; \lambda, \alpha)+f(-z ; \lambda, \alpha) \\
& =\frac{2}{\alpha+2} \phi(z)\left[1+\alpha \Phi\left(\frac{\lambda z}{\sqrt{1+\lambda^{2} z^{2}}}\right)\right]+\frac{2}{\alpha+2} \phi(z)\left[1+\alpha \Phi\left(\frac{-\lambda z}{\sqrt{1+\lambda^{2} z^{2}}}\right)\right] \\
& =2 \phi(z),
\end{aligned}
$$

which is the p.d.f. of a half normal distribution as $\phi(\cdot)$ is the p.d.f. of a standard normal variate.

Result 2.3. If $Z$ has the $\operatorname{ESCND}(\lambda, \alpha)$ then $Z^{2}$ follows chi-square distribution with one degree of freedom. 
Proof. Let $Z_{3}=Z^{2}$. The p.d.f. $f_{3}\left(z_{3} ; \lambda, \alpha\right)$ of $Z_{3}$ is the following, for $z_{3}>0$.

$$
\begin{aligned}
f_{3}\left(z_{3} ; \lambda, \alpha\right) & =\phi\left(\sqrt{z_{3}}, \lambda, \alpha\right)\left|\frac{d z}{d z_{3}}\right|+\phi\left(-\sqrt{z_{3}}, \lambda, \alpha\right)\left|\frac{d z}{d z_{3}}\right| \\
& =\frac{2 \phi\left(\sqrt{z_{3}}\right)}{(\alpha+2)\left(2 \sqrt{z_{3}}\right)}\left[1+\alpha \Phi\left(\frac{\lambda \sqrt{z_{3}}}{\sqrt{1+\lambda^{2} z_{3}}}\right)\right]+\frac{2 \phi\left(-\sqrt{z_{3}}\right)}{(\alpha+2)\left(2 \sqrt{z_{3}}\right)}\left[1+\alpha \Phi\left(\frac{-\lambda \sqrt{z_{3}}}{\sqrt{1+\lambda^{2} z_{3}}}\right)\right] \\
& =\left(1 / \sqrt{z_{3}}\right) \phi\left(\sqrt{z_{3}}\right) \\
& =(1 / \sqrt{2 \pi}) z_{3}^{\frac{1}{2}-1} e^{-\frac{z_{3}}{2}},
\end{aligned}
$$

which is the p.d.f. of a chi-square variate with one degrees of freedom.

Result 2.4. The characteristic function, $\psi(t)$ of a random variable $Z$ following $\operatorname{ESCND}(\lambda, \alpha)$ with p.d.f. (2.1) is the following, for any $t \in R$ and $i=\sqrt{-1}$.

$$
\psi(t)=\frac{2}{\alpha+2} e^{-\frac{t^{2}}{2}}\left\{1+\alpha E\left(\Phi\left(\frac{\lambda(W+i t)}{\sqrt{1+\lambda^{2}(W+i t)^{2}}}\right)\right)\right\}
$$

where $W$ is a standard normal variate.

Proof. Let $Z$ follows $\operatorname{ESCND}(\lambda, \alpha)$ with p.d.f. (2.1). By the definition of characteristic function, for any $t \in R$ and $i=\sqrt{-1}$, we have

$$
\begin{aligned}
\psi(t) & =E\left(e^{i t Z}\right) \\
& =\frac{2}{\alpha+2}\left[\int_{-\infty}^{\infty} e^{i t z} \phi(z) d z+\alpha \int_{-\infty}^{\infty} e^{i t z} \frac{1}{\sqrt{2 \pi}} e^{-\frac{z^{2}}{2}} \Phi\left(\frac{\lambda z}{\sqrt{1+\lambda^{2} z^{2}}}\right) d z\right] \\
& =\frac{2}{\alpha+2} e^{-\frac{t^{2}}{2}}\left[1+\alpha \int_{-\infty}^{\infty} \phi(z-i t) \Phi\left(\frac{\lambda z}{\sqrt{1+\lambda^{2} z^{2}}}\right) d z\right]
\end{aligned}
$$

On substituting $z-i t=w, \psi(t)$ reduces to

$$
\psi(t)=\frac{2}{\alpha+2} e^{-\frac{t^{2}}{2}}\left[1+\alpha \int_{-\infty}^{\infty} \phi(w) \Phi\left(\frac{\lambda(w+i t)}{\sqrt{1+\lambda^{2}(w+i t)^{2}}}\right) d w\right]
$$

which leads to $(2.2)$.

Result 2.5. The c.d.f. of a random variable $Z$ following $\operatorname{ESCND}(\lambda, \alpha)$ is

$$
F(z ; \lambda, \alpha)=\Phi(z)-\frac{2 \alpha}{\alpha+2} \xi\left(z ; \lambda t / \sqrt{1+\lambda^{2} t^{2}}\right),
$$

where for any $a, b \in R$,

$$
\xi(a, b)=\int_{a}^{\infty} \phi(t) \int_{0}^{b} \phi(u) d u d t
$$




\section{Moments}

By Result 2.3, the even moments of the $\operatorname{ESCND}(\lambda, \alpha)$ and those of the standard normal distribution are identical. So, for computing odd moments, we develop the following result. Result 3.1. If $Z$ follows $\operatorname{ESCND}(\lambda, \alpha)$, then for $k=0,1,2, \ldots$

$$
E\left(Z^{2 k+1}\right)=\frac{\left[2^{k+1} \Gamma(k+1)+\alpha \sqrt{2 \pi} \Lambda_{k}(\lambda)\right]}{(\alpha+2) \sqrt{2 \pi}}
$$

in which for $\lambda \in R$,

$$
\Lambda_{k}(\lambda)=\int_{0}^{\infty} \frac{u^{k}}{\sqrt{2 \pi}} e^{-\frac{u^{2}}{2}} \Phi\left(\frac{\lambda \sqrt{u}}{\sqrt{1+\lambda^{2} u}}\right) d u
$$

Proof. By definition,

$$
E\left(Z^{2 k+1}\right)=\int_{-\infty}^{\infty} z^{2 k+1} \frac{2}{\alpha+2} \phi(z)\left[1+\alpha \Phi\left(\frac{\lambda z}{\sqrt{1+\lambda^{2} z^{2}}}\right)\right] d z
$$

On substituting $z^{2}=u$, we get

$$
\begin{aligned}
E\left(Z^{2 k+1}\right) & =\int_{0}^{\infty} u^{k+\frac{1}{2}} \frac{2}{\alpha+2} \frac{1}{\sqrt{2 \pi}} e^{-\frac{u^{2}}{2}}\left[1+\alpha \Phi\left(\frac{\lambda \sqrt{u}}{\sqrt{1+\lambda^{2} u}}\right)\right] \frac{1}{2 \sqrt{u}} d u \\
& =\frac{1}{\alpha+2}\left[\int_{0}^{\infty} u^{k} \frac{e^{-\frac{u^{2}}{2}}}{\sqrt{2 \pi}} d u+\alpha \int_{0}^{\infty} u^{k} \frac{e^{-\frac{u^{2}}{2}}}{\sqrt{2 \pi}} \Phi\left(\frac{\lambda \sqrt{u}}{\sqrt{1+\lambda^{2} u}}\right) d u\right],
\end{aligned}
$$

which implies (3.1).

Result 3.2. The p.d.f. of the $\operatorname{ESCND}(\lambda, \alpha)$ is $\log$ concave for the following cases, in which

$$
\kappa(\lambda)=\frac{\alpha \lambda_{1}^{2} \phi^{\prime}(d)}{[1+\alpha \Phi(d)]\left[1+\lambda^{2} z^{2}\right]^{3}}
$$

and

$$
\zeta(\lambda)=\frac{\alpha \lambda^{3} z}{[1+\alpha \Phi(d)]\left[1+\lambda^{2} z^{2}\right]^{5 / 2}},
$$

with $d=\lambda z / \sqrt{1+\lambda^{2} z^{2}}$.

(i) For $\alpha>0, \lambda z>0$ if $\kappa(\lambda) \leq 1$,

(ii) $\alpha<0, \lambda z<0$ if $\kappa(\lambda) \leq 1$,

(iii) $\alpha>0, \lambda z<0$ if $\kappa(\lambda)+3 \zeta(\lambda) \leq 1$ and 
(iv) $\alpha \in(-1,0), \lambda z>0$ if $\kappa(\lambda)+3 \zeta(\lambda) \leq 1$.

Proof. Taking logarithm on both sides of the p.d.f. of the $\operatorname{ESCND}(\lambda, \alpha)$ given in (2.1) and differentiating twice with respect to $z$, we get the following, in which

$$
\eta(\lambda)=\frac{\alpha^{2} \lambda^{2} \phi(d)^{2}}{[1+\alpha \phi(d)]^{2}\left[1+\lambda^{2} z^{2}\right]^{3}},
$$

$\kappa(\lambda)$ and $\zeta(\lambda)$ are as given in (3.2) and (3.3)

$$
\frac{d^{2}}{d z^{2}}[\log (f(z ; \lambda, \alpha))]=-1-\eta(\lambda)+\kappa(\lambda)-3 \zeta(\lambda)
$$

Note that $\eta(\lambda)$ is positive for all $\alpha, \lambda z \in R$ and $\alpha /\{1+\alpha \Phi(d)\}$ is positive for any $\lambda z \in R$, for $\alpha>0$ and negative for all $\alpha<0, \lambda z<0$ or $\alpha \in(-1,0), \lambda z>0$. Thus, $\zeta(\lambda)$ given in (3.3) is positive for $\alpha>0, \lambda z>0$ or $\alpha<0, \lambda z<0$. Hence (3.4) will be negative for $\alpha>0$, $\lambda z>0$ or $\alpha<0, \lambda z<0$ provided $\kappa(\lambda) \leq 1$.

If $\alpha>0$ and $\lambda z<0$, then $\zeta(\lambda)$ is negative and in this case (3.4) will be negative only when $\kappa(\lambda)-3 \zeta(\lambda) \leq 1$. Also, if $\alpha \in(-1,0)$ and $\lambda z>0$, then $\zeta(\lambda)$ will be negative and hence (3.4) will be negative only when $\kappa(\lambda)-3 \zeta(\lambda) \leq 1$. Hence $f(z ; \lambda, \alpha)$ is log-concave for those case as mentioned in (i), (ii), (iii) and (iv).

Result 3.3. The p.d.f. of the $\operatorname{ESCND}(\lambda, \alpha)$ is unimodal at least for the following cases:

(i) For $\alpha>0, \lambda z>0$ if $\kappa(\lambda) \leq 1$,

(ii) $\alpha<0, \lambda z<0$ if $\kappa(\lambda) \leq 1$,

(iii) $\alpha>0, \lambda z<0$ if $\kappa(\lambda)+3 \zeta(\lambda) \leq 1$ and

(iv) $\alpha \in(-1,0), \lambda z>0$ if $\kappa(\lambda)+3 \zeta(\lambda) \leq 1$.

Result 3.4. The p.d.f. of the $\operatorname{ESCND}(\lambda, \alpha)$ is plurimodal at least for the following cases:

(i) For $\alpha>0, \lambda z>0$ if $\kappa(\lambda)>1+\eta(\lambda)+3 \zeta(\lambda)$,

(ii) $\alpha<0, \lambda z<0$ if $\kappa(\lambda)>1+\eta(\lambda)+3 \zeta(\lambda)$,

(iii) $\alpha>0, \lambda z<0$ if $\kappa(\lambda)+3 \zeta(\lambda)>1+\eta(\lambda)$ and

(iv) $\alpha \in(-1,0), \lambda z>0$ if $\kappa(\lambda)+3 \zeta(\lambda)>1+\eta(\lambda)$.

\section{Reliability Aspects}

Here we derive explicit expressions for certain reliability measures of the $\operatorname{ESCND}(\lambda, \alpha)$ such as reliability function, failure rate and mean residual life function. 
Result 4.1. The reliability function $R(t ; \lambda, \alpha)$ of the $\operatorname{ESCND}(\lambda, \alpha)$ is given by

$$
R(t ; \lambda, \alpha)=1-\Phi(t)+\frac{2 \alpha}{\alpha+2} \xi\left(t ; \frac{\lambda t}{\sqrt{1+\lambda^{2} t^{2}}}\right),
$$

where $\xi\left(t ; \lambda t / \sqrt{1+\lambda^{2} t^{2}}\right)$ is as defined in (2.4).

The proof follows from the definition of reliability function and Result 2.5.

Result 4.2. The failure rate $r(t ; \lambda, \alpha)$ of the $\operatorname{ESCND}(\lambda, \alpha)$ is given by

$$
r(t ; \lambda, \alpha)=\frac{2 \phi(t)\left[1+\alpha \Phi\left(\frac{\lambda t}{\sqrt{1+\lambda^{2} t^{2}}}\right)\right]}{(\alpha+2)[1-\Phi(t)]+2 \alpha \xi\left(t ; \frac{\lambda t}{\sqrt{1+\lambda^{2} t^{2}}}\right)}
$$

The proof follows from the definition of failure rate and Result 2.5.

Note that when $\alpha>0$, the reliability function $R(t ; \lambda, \alpha)$ is a decreasing function of $\mathrm{t}$ where as the failure rate $r(t ; \lambda, \alpha)$ is an increasing function of $\mathrm{t}$.

Result 4.3. The mean residual life function $M(t ; \lambda, \alpha)$ of $\operatorname{ESCND}(\lambda, \alpha)$ is the following.

$$
M(t ; \lambda, \alpha)=\frac{2}{(\alpha+2) R(t ; \lambda, \alpha) \sqrt{2 \pi}}\left[e^{-\frac{t^{2}}{2}}+\alpha e^{-\frac{t^{2}}{2}} \Phi\left(\frac{\lambda t}{\sqrt{1+\lambda^{2} t^{2}}}\right)+\alpha \sqrt{2 \pi} \xi^{*}(t ; \lambda)\right]-t
$$

in which

$$
\xi^{*}(t ; \lambda)=\int_{t}^{\infty} \phi(z)\left[\frac{d}{d z}\left(\int_{0}^{\frac{\lambda z}{\sqrt{1+\lambda^{2} z^{2}}}} \phi(u) d u\right)\right] d z
$$

Proof. By definition, the mean residual life function $M R L F(t)$ of $\mathrm{Z}$ is

$$
M R L F(t)=E(Z-t \mid Z>t)=E(Z \mid Z>t)-t
$$

where

$$
\begin{aligned}
E(Z \mid Z>t) & =\frac{1}{(\alpha+2) R(t ; \lambda, \alpha)} \int_{t}^{\infty}\left[2 z \phi(z) d z+2 \alpha z \phi(z) \Phi\left(\frac{\lambda z}{\sqrt{1+\lambda^{2} z^{2}}}\right)\right] d z \\
& =\frac{2}{(\alpha+2) R(t ; \lambda, \alpha) \sqrt{2 \pi}}\left[e^{-\frac{t^{2}}{2}}+\alpha e^{-\frac{t^{2}}{2}} \Phi\left(\frac{\lambda t}{\sqrt{1+\lambda^{2} t^{2}}}\right)+\alpha \sqrt{2 \pi} \xi^{*}(t ; \lambda)\right]
\end{aligned}
$$

which implies (4.1).

The functions $R(t ; \lambda, \alpha), r(t ; \lambda, \alpha)$ and $M(t ; \lambda, \alpha)$ are equivalent in the sense that if one of them is given the other two can be uniquely determined. 


\section{Location Scale Extension and Properties}

In this section, we present the definition of the location scale extension of the $\operatorname{ESCND}(\lambda, \alpha)$ and discuss some of its important properties.

Definition 5.1. The location-scale extension of a random variable $Z$ following the $\operatorname{ESCND}(\lambda, \alpha)$ is defined as the distribution of $X=\mu+\sigma Z$, whose p.d.f. is as given below, in which $\lambda \in R$, $\alpha \geq-1$.

$$
g(x ; \mu, \sigma, \lambda, \alpha)=\frac{2}{(\alpha+2) \sigma} \phi\left(\frac{x-\mu}{\sigma}\right)\left\{1+\alpha \Phi\left[\frac{\lambda(x-\mu)}{\sqrt{\sigma^{2}+\lambda^{2}(x-\mu)^{2}}}\right]\right\},
$$

where $\mu \in R$ and $\sigma>0$ are respectively the location and scale parameters. A distribution with p.d.f. (5.1) we call the extended generalized skew curved normal distribution and is denoted as $\operatorname{EGSCND}(\mu, \sigma ; \lambda, \alpha)$.

Clearly

1. when $\alpha=0$ and/or when $\lambda=0$, the p.d.f. given in (5.1) reduces to that of normal distribution $N\left(\mu, \sigma^{2}\right)$ and

2. when $\alpha=-1$, the p.d.f. given in (5.1) reduces to that of the location scale extended form of skew curved normal distribution with parameters $\mu, \sigma$ and $-\lambda$.

Now we obtain the following properties of the $\operatorname{EGSCND}(\mu, \sigma ; \lambda, \alpha)$.

Result 5.1. If the conditional distribution of $U$ given $V=v$ follows $\operatorname{EGSCND}(\mu, \sigma ; v, \alpha)$ and $V$ follows $N\left(0, \sigma_{1}^{2}\right)$ then $U$ follows $N\left(\mu, \sigma^{2}\right)$ and the conditional distribution of $T_{1}=\sigma_{1}^{-1} V$ given $U_{1}=u$ follows $\operatorname{EGSCND}\left(0, \sigma ; \sigma_{1}(u-\mu), \alpha\right)$.

Proof. Let $q(u, v), q_{10}(u / v)$ and $q_{2}(v)$ denote respectively the joint p.d.f of $U$ and $V$, the conditional p.d.f. of $U$ given $V$ and the marginal p.d.f. of $V$.

Then the marginal p.d.f. of $U$ is

$$
\begin{aligned}
q_{1}(u) & =\int_{-\infty}^{\infty} q_{10}(u / v) q_{2}(v) d v \\
& =\frac{2}{(\alpha+2) \sigma \sigma_{1}} \phi\left(\frac{u-\mu}{\sigma}\right) \int_{-\infty}^{\infty}\left\{1+\alpha \Phi\left[\frac{v(u-\mu)}{\sqrt{\sigma^{2}+v^{2}(u-\mu)^{2}}}\right]\right\} \phi\left(\frac{v}{\sigma_{1}}\right) d v
\end{aligned}
$$

On substituting $v=\sigma_{1} t$ in (5.2) we get

$$
\begin{aligned}
q_{1}(u) & =\frac{1}{(\alpha+2)} \frac{1}{\sigma} \phi\left(\frac{u-\mu}{\sigma}\right)\left[2+\alpha \int_{-\infty}^{\infty} 2 \Phi\left(\frac{\sigma_{1}(u-\mu) t}{\sqrt{\sigma^{2}+\sigma_{1}^{2}(u-\mu)^{2} t^{2}}}\right) \phi(t) d t\right] \\
& =\frac{1}{\sigma} \phi\left(\frac{u-\mu}{\sigma}\right)
\end{aligned}
$$


since the integrant in the second term of the right hand side expression of (5.3) is the p.d.f. of a location scale extension of the skew curved normal distribution. Thus, (5.3) shows that $U$ follows $N\left(\mu, \sigma^{2}\right)$.

Now for $v \in R$, the conditional p.d.f. $q_{01}(v \mid u)$ of $V$ given $U=u$ is

$$
\begin{aligned}
q_{01}(v \mid u) & =\frac{q(u, v)}{q_{1}(u)} \\
& =\frac{q_{10}(u \mid v) q_{2}(v)}{q_{1}(u)} \\
& =\frac{2 \phi\left(\frac{u-\mu}{\sigma}\right)}{(\alpha+2) \sigma \sigma_{1}}\left\{1+\alpha \Phi\left[\frac{v(u-\mu)}{\sqrt{\sigma^{2}+v^{2}(u-\mu)^{2}}}\right]\right\} \frac{\phi\left(v / \sigma_{1}\right)}{(1 / \sigma) \phi\left(\frac{u-\mu}{\sigma}\right)} .
\end{aligned}
$$

On substituting $t=\sigma_{1}^{-1} v$, we get

$$
q_{01}\left(t \sigma_{1} \mid u\right)=\frac{2}{(\alpha+2) \sigma}\left\{1+\alpha \Phi\left[\frac{t \sigma_{1}(u-\mu)}{\sqrt{\sigma^{2}+t^{2} \sigma_{1}^{2}(u-\mu)^{2}}}\right]\right\} \phi(t),
$$

which is the p.d.f. of the $\operatorname{EGSCND}\left(0, \sigma ; \sigma_{1}(u-\mu), \alpha\right)$.

Result 5.2. Assume that: (i) the conditional distribution of $U$ given $V_{1}=v$ and $V_{2}=v$ follows $E G S C N D(\mu, \sigma ; v, \alpha)$, (ii) the conditional distribution of $V_{1}$ given $V_{2}=v$ follows $N\left(0, \sigma_{1}^{2}\right)$, (iii) $V_{1}$ and $V_{2}$ are independent. Then (i) $U$ and $V_{2}$ are independent, (ii) $U$ follows $N\left(\mu, \sigma^{2}\right)$ and (iii) the conditional distribution of $V_{1}$ given $U=u, T_{2}=t$ follows $\operatorname{EGSCND}\left(0, \sigma ; \sigma_{1}(u-\mu), \alpha\right)$, where $T_{2}=\sigma_{1}^{-1} V_{2}$.

Proof. In order to prove that $U$ and $V_{2}$ are independent it is enough to show that the conditional distribution of $U$ given $V_{2}$ is independent of $v$. For $u \in R$, the conditional p.d.f. of $U$ given $V_{2}=v$ is

$$
\begin{aligned}
g_{U \mid V_{2}}(u) & =\int_{-\infty}^{\infty} g_{U \mid V_{1}, V_{2}}(u) g_{V_{1}}(v) d v \\
& =\int_{-\infty}^{\infty} \frac{2}{(\alpha+2) \sigma} \phi\left(\frac{u-\mu}{\sigma}\right)\left\{1+\alpha \Phi\left[\frac{v(u-\mu)}{\sqrt{\sigma^{2}+v^{2}(u-\mu)^{2}}}\right]\right\} \frac{1}{\sigma_{1}} \phi\left(\frac{v}{\sigma_{1}}\right) d v
\end{aligned}
$$

On substituting $\frac{v}{\sigma_{1}}=t$ in (5.4) we get

$$
\begin{aligned}
g_{U \mid V_{2}}(u) & =\frac{1}{(\alpha+2) \sigma} \phi\left(\frac{u-\mu}{\sigma}\right)\left[2+\alpha \int_{-\infty}^{\infty} \Phi\left(\frac{\sigma_{1} t(u-\mu)}{\sqrt{\sigma^{2}+\sigma_{1}^{2} t^{2}(u-\mu)^{2}}}\right) \phi(t) d t\right] \\
& =\frac{1}{\sigma} \phi\left(\frac{u-\mu}{\sigma}\right)
\end{aligned}
$$


which is independent on $v$. This shows that $U$ and $V_{2}$ are independent and $U$ follows $N\left(\mu, \sigma^{2}\right)$.

Now, for $v \in R$,

$$
\begin{aligned}
g_{V_{1} \mid U, V_{2}}(v) & =\frac{g_{U, V_{1} \mid V_{2}}(u, v)}{g_{U \mid V_{2}}(u)} \\
& =\frac{2 \phi\left(v / \sigma_{1}\right)}{(\alpha+2) \sigma_{1}}\left\{1+\alpha \Phi\left[\frac{v(u-\mu)}{\sqrt{\sigma^{2}+v^{2}(u-\mu)^{2}}}\right]\right\} .
\end{aligned}
$$

If we take $v=\sigma_{1} t$, then (5.5) leads to

$$
g_{V_{1} \mid U, T_{2}}\left(\sigma_{1} t\right)=\frac{2 \phi(t)}{(\alpha+2) \sigma_{1}}\left\{1+\alpha \Phi\left[\frac{\sigma_{1} t(u-\mu)}{\sqrt{\sigma^{2}+\sigma_{1}^{2} t^{2}(u-\mu)^{2}}}\right]\right\}
$$

which implies that $\left(V_{1} \mid U=u, T_{2}=t\right)$ follows $\operatorname{EGSCND}\left(0, \sigma ; \sigma_{1}(u-\mu), \alpha\right)$.

Result 5.3. If the conditional distribution of $U$ given $V=v$ follows $\operatorname{GMNSND}(v, \alpha)$ and $V$ follows $N\left(\lambda, \lambda^{2}\right)$ then $U$ follows $\operatorname{ESCND}(\lambda, \alpha)$.

Proof. The proof follows from the similar arguments we made in the proof of Result 5.1.

\section{Maximum Likelihood Estimation}

In this section we discuss the maximum likelihood estimation of the parameters of the $\operatorname{EGSCND}(\mu, \sigma ; \lambda, \alpha)$. Let $X_{1}, X_{2}, \ldots, X_{n}$ be a random sample from a population following $\operatorname{EGSCND}(\mu, \sigma ; \lambda, \alpha)$ with p.d.f. (5.1). Then the likelihood function of the random sample is

$$
L=\left(\frac{2}{\sqrt{2 \pi}}\right)^{n} e^{-\sum_{i=1}^{n} \frac{\left(x_{i}-\mu\right)^{2}}{2 \sigma^{2}}}\left\{(\alpha+2)^{n} \sigma^{n}\right\}^{-1} \prod_{i=1}^{n}\left[1+\alpha \Phi\left(d_{i}\right)\right],
$$

where $d_{i}=\lambda\left(x_{i}-\mu\right) / \sqrt{\sigma^{2}+\lambda^{2}\left(x_{i}-\mu\right)^{2}}$. Taking logarithm on both sides, we get

$$
\log L=n \log \left(\frac{2}{\sqrt{2 \pi}}\right)-\sum_{i=1}^{n} \frac{\left(x_{i}-\mu\right)^{2}}{2 \sigma^{2}}-n \log (\alpha+2)-n \log \sigma+\sum_{i=1}^{n} \log \left[1+\alpha \Phi\left(d_{i}\right)\right]
$$


On differentiating (6.1) with respect to $\mu, \sigma^{2}, \lambda$, and $\alpha$ respectively and equating to zero, we get the following normal equations:

$$
\begin{aligned}
& \frac{\partial \log L}{\partial \mu}=0 \Rightarrow \sum_{i=1}^{n} \frac{\left(x_{i}-\mu\right)}{\sigma^{2}}=\sum_{i=1}^{n} \frac{\alpha \phi\left(d_{i}\right) \lambda \sigma^{2}}{\left[1+\alpha \Phi\left(d_{i}\right)\right]}\left[\sigma^{2}+\lambda^{2}\left(x_{i}-\mu\right)^{2}\right]^{-\frac{3}{2}}, \\
& \frac{\partial \log L}{\partial \sigma^{2}}=0 \Rightarrow \frac{n}{2 \sigma^{2}}=\sum_{i=1}^{n} \frac{\left(x_{i}-\mu\right)^{2}}{2 \sigma^{4}}-\sum_{i=1}^{n} \frac{\alpha \phi\left(d_{i}\right) \lambda\left(x_{i}-\mu\right)}{2\left\{1+\alpha \Phi\left(d_{i}\right)\right\}}\left[\sigma^{2}+\lambda^{2}\left(x_{i}-\mu\right)^{2}\right]^{-\frac{3}{2}}, \\
& \frac{\partial \log L}{\partial \lambda}=0 \Rightarrow \sum_{i=1}^{n} \frac{\alpha \sigma^{2} \phi\left(d_{i}\right)}{1+\alpha \Phi\left(d_{i}\right)}\left(\frac{\left(x_{i}-\mu\right)}{\left(\sigma^{2}+\lambda^{2}\left(x_{i}-\mu\right)^{2}\right)^{\frac{3}{2}}}\right)=0 \text { and } \\
& \frac{\partial \log L}{\partial \alpha}=0 \Rightarrow \frac{-n}{\alpha+2}+\sum_{i=1}^{n} \frac{\Phi\left(d_{i}\right)}{1+\alpha \Phi\left(d_{i}\right)}=0 .
\end{aligned}
$$

If we put

$$
\frac{\phi\left(d_{i}\right)\left(\sigma^{2}+\lambda^{2}\left(x_{i}-\mu\right)^{2}\right)^{-\frac{3}{2}}}{1+\alpha \Phi\left(d_{i}\right)}=W\left(x_{i}\right) \text { and } \frac{\Phi\left(d_{i}\right)}{1+\alpha \Phi\left(d_{i}\right)}=V\left(x_{i}\right)
$$

in equations (6.2) to (6.5) to get

$$
\begin{aligned}
\sum_{i=1}^{n} \frac{\left(x_{i}-\mu\right)}{\sigma^{2}}-\lambda \sigma^{2} \sum_{i=1}^{n} \alpha W\left(x_{i}\right) & =0 \\
\sum_{i=1}^{n} \frac{\left(x_{i}-\mu\right)^{2}}{\sigma^{4}}-\alpha \lambda \sum_{i=1}^{n} W\left(x_{i}\right)\left(x_{i}-\mu\right) & =\frac{n}{\sigma^{2}} \\
\sum_{i=1}^{n} \alpha \sigma^{2}\left(x_{i}-\mu\right) W\left(x_{i}\right) & =0 \\
\frac{n}{\alpha+2}-\sum_{i=1}^{n} V\left(x_{i}\right) & =0 .
\end{aligned}
$$

Solving the equations from (6.6) to (6.9) using mathematical software one can obtain the maximum likelihood estimates of the parameters of the $\operatorname{EGSCND}(\mu, \sigma ; \lambda, \alpha)$, provided the following inequalities hold good.

$$
\begin{aligned}
\sum_{i=1}^{n} \frac{\partial}{\partial \mu} W\left(x_{i}\right) & <\frac{-1}{\alpha \lambda \sigma^{4}} \\
\sum_{i=1}^{n}\left(2 W\left(x_{i}\right)\left(x_{i}-\mu\right)+\sigma^{2} \frac{\partial}{\partial \sigma^{2}} W\left(x_{i}\right)\left(x_{i}-\mu\right)\right) & <\frac{-n}{\alpha \lambda \sigma^{2}} \\
\frac{\sum_{i=1}^{n} x \frac{\partial}{\partial \lambda} W\left(x_{i}\right)}{\sum_{i=1}^{n} \frac{\partial}{\partial \lambda} W\left(x_{i}\right)} & <\mu \text { and } \sum_{i=1}^{n} \frac{\partial}{\partial \alpha} V\left(x_{i}\right)<\frac{-n}{(\alpha+2)^{2}} .
\end{aligned}
$$




\section{An Application}

For carrying out a numerical illustration, here we consider the data matrices related to milk production of 28 cows taken from Bhuyan (2005), which we presented as Dataset and is given in the following Table 1. The variable under study is the daily milk production

in kilogram, the variable recorded for three times in milking cows. For model comparison

Table 1: Data set

\begin{tabular}{cccccccccccccc}
\hline 34.6 & 27.7 & 29.2 & 25.3 & 27.6 & 37.9 & 32.6 & 32 & 30.7 & 29.6 & 38.3 & 32.9 & 30.8 & 32.2 \\
32.9 & 28.1 & 33.9 & 28.6 & 28.1 & 35.9 & 34.8 & 40.3 & 30.9 & 34.4 & 19.8 & 25.8 & 37.3 & 32.4 \\
\hline
\end{tabular}

we have calculated the information criteria - AIC, BIC and the AICc. The numerical results obtained are presented in Table 1 , which includes the estimated values of the parameters, log-likelihood (l), $A I C, B I C$ and $A I C c$ for different models such as $N(\mu, \sigma)$, $\operatorname{ESND}(\mu, \sigma, \lambda), \operatorname{ESCND}(\mu, \sigma, \lambda), \operatorname{EGMNSND}(\mu, \sigma, \lambda, \alpha)$ and $\operatorname{EGSCND}(\mu, \sigma, \lambda)$. From

Table 2: Estimated values of the parameters, $l, A I C, B I C$ and $A I C c$ for the distributions $N(\mu, \sigma), \operatorname{ESND}(\mu, \sigma, \lambda), \operatorname{ESCND}\left(\mu, \sigma, \lambda_{1}, \lambda_{2}\right), \operatorname{EGMNSND}(\mu, \sigma, \lambda, \alpha)$ and $\operatorname{EGSCND}\left(\mu, \sigma, \lambda_{1}, \lambda_{2}, \alpha\right)$

\begin{tabular}{cccccc}
\hline $\begin{array}{c}\text { Parameters } \\
\text { criteria }\end{array}$ & $\begin{array}{c}N \\
(\mu, \sigma)\end{array}$ & $\begin{array}{c}\text { ESND } \\
(\mu, \sigma, \lambda)\end{array}$ & $\begin{array}{c}\text { ESCND } \\
(\mu, \sigma, \lambda)\end{array}$ & $\begin{array}{c}\text { EGMNSND } \\
(\mu, \sigma, \lambda, \alpha)\end{array}$ & $\begin{array}{c}\text { EGSCND } \\
(\mu, \sigma, \lambda, \alpha)\end{array}$ \\
\hline$\mu$ & 31.59286 & 32.95 & 31.59 & 31.596 & 31.95 \\
$\sigma$ & 4.46 & 4.58 & 4.38 & 4.38 & 4.62 \\
$\lambda$ & - & -0.39 & 0.562 & 0.07 & 0.394 \\
$\alpha$ & - & - & - & 0.485 & 2.27 \\
$l$ & -81.11 & -81.08 & -81.10 & -78.01 & -63.32 \\
$A I C$ & 166.23 & 168.17 & 170.21 & 164.02 & 136.65 \\
$B I C$ & 168.9 & 172.17 & 175.54 & 169.36 & 143.3 \\
$A I C c$ & 166.7 & 169.17 & 171.95 & 165.77 & 139.37 \\
\hline
\end{tabular}

Table 2 and Figure 3, it can be seen the $\operatorname{EGSCND}(\mu, \sigma, \lambda, \alpha)$ gives a better fit to the data considered here compared to the existing models- $N(\mu, \sigma), \operatorname{ESN} D(\mu, \sigma, \lambda), \operatorname{ESCND}(\mu, \sigma, \lambda)$ and $\operatorname{EGMNSND}(\mu, \sigma, \lambda, \alpha)$. From Table 2 it can also be observe that the EGMNSND have not given better fit to the data set mainly due to the moderate value of $\lambda$, while the $E G S C N D$ overcomes this limitation. 
Figure 3: Plots showing the c.d.f. of various models: $N, E S N D, E S C N D, E G M N S N D$, $E G S C N D$ and empirical c.d.f.

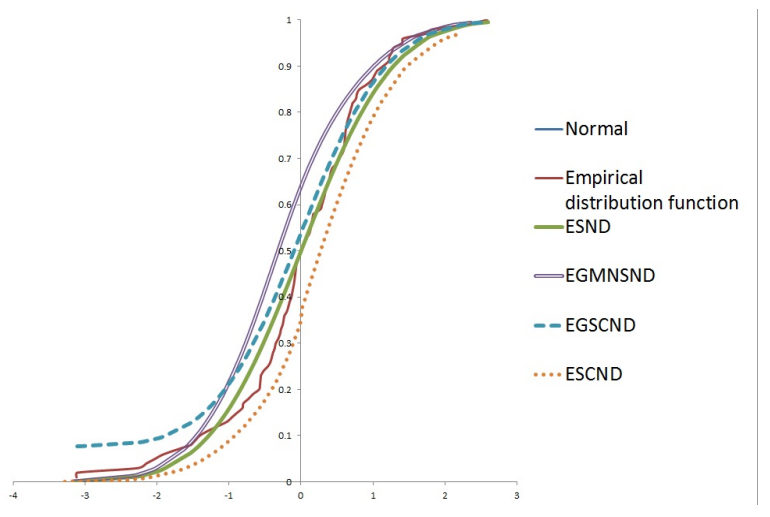

\section{Summary and Conclusion}

Through this paper we introduce and study a new family of asymmetric normal distributions namely "the extended skew curved normal distribution (ESCND)", that exhibits a better behavior compared to several existing asymmetric normal models, particularly at the tails with smaller mass. The ESCND is obtained as a generalized mixture of the standard normal distribution and the skew curved normal distribution of Arellano-Valle et al. (2004). A location scale extended class of the ESCND is also considered for providing more flexible classes of asymmetric as well as plurimodel normal models. Moreover, we have illustrated the relevance of the proposed model in fitting real life data sets compared to existing models in such categories.

\section{Acknowledgements}

The authors express their immense gratitude to the Editor, Associate Editor and anonymous referees for their valuable comments on an earlier version of the manuscript that greatly improved the quality and presentation of the paper.

\section{References}

Arellano-Valle, R. B., Gomez, H. W. and Quintana, F. A. (2004), "A new class of skew normal distribution", Communications in Statistics- Theory and Methods, 33(7), 1465-1480. 
Azzalini, A. (1985), "A class of distributions which includes the normal ones", Scandinavian Journal of Statistics, 12, 171-178.

Azzalini, A. (1986), "Further results on a class of distributions which includes the normal ones", Statistica XLVI, 199-208.

Azzalini, A. (2005), "The skew-normal distribution and related multivariate families", Scandinavian Journal of Statistics, 32, 159-188.

Bhuyan, K. C. (2005), "Multivariate analysis and its applications", New Central Book Agency, Kolkata, India.

Birnbaum, Z. W. (1950), "Effect of linear truncation on a multinormal population", Annals of Mathematical Statistics, 21, 272-279.

Buccianti, A. (2005), "Meaning of the $\lambda$ parameter of skew normal and log skew normal distributions in fluid geo chemistry", CODAWORK'05, October 19-21, 1-15.

Edgeworth, F. Y. (1886), "The law of error and the elimination of chance", Philosophical Magazine, 21, 308-324.

Genton, M. G., ed. (2004), "Skew-elliptical distributions and their applications: A journey beyond normality", Chapman and Hall/CRC, London.

Khamis, A., Ismail, Z., Haron, K. and Mohammed, A. T. ( 2005), "Nonlinear growth models for modeling oil palm yield growth", Journal of Mathematics and Statistics, $1(3), 225-233$.

Kumar, C. S. and Anusree, M. R. (2011), "On a generalized mixture of standard normal and skew normal distributions", Statistics and Probability Letters, 81, 1813-1821.

Nelson, L. S. (1964), "The sum of values from a normal and a truncated normal distribution", Technometrics, 6, 469-471.

ÓHagan, A. and Leonard, T. (1976), "Bayes estimation subject to uncertainty about parameter constraints", Biometrika, 63, 201-203.

Pearson, K. (1893), "Asymmetrical frequency curves", Nature, 48, 615-616.

Roberts, C. (1966), "A correlation model useful in the study of twins", Journal of American Statistical Association, 61, 1184-1190.

Weinstein, M. A. (1964), "The sum of values from a normal and a truncated normal distribution", Technometrics, 6, 104-105.

Received: May 27, 2017

Accepted: January 16, 2018 